\title{
Hipertiroidismo secundario a coriocarcinoma metastásico, reporte de caso
}

\author{
Hyperthyroidism Secondary to Metastatic Choriocarcinoma: \\ Case Report
}

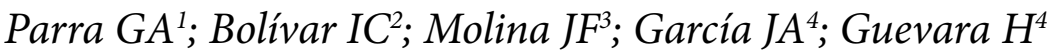

\author{
${ }^{1}$ Especialista en Medicina interna, Endocrinología. Profesor \\ Asociado UNAB, FOSCAL. \\ ${ }^{2}$ Médica Patóloga en Foscal Internacional. \\ ${ }^{3}$ Residente de Medicina interna Universidad de Cartagena \\ ${ }^{4}$ Estudiante Internado II Universidad de Pamplona
}

Fecha de recepción: 27/11/2018

Fecha de aceptación: 29/01/2019

\section{Resumen}

Aunque la enfermedad de Graves sigue siendo considerada la causa más frecuente de hipertiroidismo clínico en el embarazo, la producción desproporcionadamente alta de gonadotropina coriónica humana (HCG) como ocurre en la enfermedad trofoblástica gestacional (ETG), es otra de las posibles causas, debido a la analogía entre ésta (HCG) y la TSH. Presentamos el caso de una paciente de 23 años que consulta por presentar dolor pélvico de moderada intensidad, síntomas respiratorios, taquicardia, temblor, astenia y pérdida de peso; a quien se le diagnostica coriocarcinoma metastásico a pulmón, con tirotoxicosis asociada, por lo que requirió manejo quimioterapéutico y antitiroideo. Se presenta este caso, por ser el hipertiroidismo secundario a coriocarcinoma una patología poco frecuente pero que debe diagnosticarse y tratarse oportunamente, ya que de eso depende el pronóstico del paciente.

Palabras clave: hipertiroidismo, tirotoxicosis, coriocarcinoma, enfermedad trofoblástica gestacional, neoplasia trofoblástica gestacional.

\section{Abstract}

Although Graves' disease continues to be considered the most frequent cause of clinical hyperthyroidism in pregnancy, the disproportionately high production of human chorionic gonadotropin (HCG) as occurs in gestational trophoblastic disease (TSG), is another possible cause, due to analogy between this (HCG) and TSH. We present the case of a 23-year-old patient who complained of pelvic pain of moderate intensity, respiratory symptoms, tachycardia, tremor, asthenia and weight loss; who was diagnosed with metastatic choriocarcinoma to the lung, with associated thyrotoxicosis, requiring chemotherapeutic and antithyroid management. This case is presented, since hyperthyroidism secondary to choriocarcinoma is a rare pathology but it must be diagnosed and treated opportunely since it depends on the patient's prognosis.

Keywords: hyperthyroidism, thyrotoxicosis, choriocarcinoma, gestational trophoblastic disease, gestational trophoblastic neoplasia.

\section{Introducción}

La enfermedad trofoblástica gestacional (ETG) es una complicación rara del embarazo, que va desde un embarazo molar a un coriocarcinoma. Ésta ocasionalmente se complica con hipertiroidismo, como consecuencia del mimetismo entre la gonadotropina coriónica humana (HCG) y la hormona estimulante de la tiroides (TSH), de ahí que una vez la ETG se trata con éxito y consecuentemente, los niveles de HCG se normalizan y el hipertiroidismo normalmente se resuelve $\mathrm{e}^{(2,8)}$.

A continuación, presentaremos un caso clínico en el que se evidencia la relación entre el hipertiroidismo y la enfermedad trofoblástica gestacional (coriocarcinoma).

\section{Reporte de caso}

Mujer de 23 años de edad con antecedente de legrado obstétrico por embarazo molar, 15 días antes; consulta por presentar dolor pélvico de moderada intensidad, disnea con deterioro de clase funcional, temblor, pérdida de peso (14 kg en cuatro meses), tos seca de predominio nocturno y astenia.

Historia de aborto espontáneo de 16 semanas en el año 2017 y embarazo ectópico con salpingooforectomía izquierda en mayo del mismo año. Al examen físico se encuentra taquicárdica (FC: $110 \mathrm{lpm}$ ), con signos de dificultad respiratoria y dolor a la palpación profunda en fosa iliaca derecha. La BHCG cuantitativa es $>100.000 \mathrm{UI} / \mathrm{ml}$, TSH $<0,005 \mu \mathrm{UI} / \mathrm{L}$ (VR. 0.27-4,20 $\mu \mathrm{UI} / \mathrm{L}$ ) y niveles de T4 libre en 5,67 ng/dl (VR. 0,81-1,52 ng/dl). 
Estudios: Ecografía transvaginal con múltiples quistes anecoicos. Ecografía de tiroides normal. Radiografía de tórax (figura 1) donde se evidencian opacidades algodonosas en lóbulos inferiores. TAC de tórax con múltiples micronódulos de aspecto de árbol en gemación en ambos campos pulmonares (figura 2, 3); TAC de abdomen con imágenes quísticas multilobuladas con escasa captación, sin componente sólido (figura 4).

El diagnóstico diferencial por las imágenes hace necesario descartar patología infecciosa (TBC) vs. enfermedad metastásica.

Estudios adicionales: PPD y BK seriada de esputo negativos; patología de biopsia y lavado broncoalveolar, reporta tumor maligno mal diferenciado con necrosis y hemorragia compatible con coriocarcinoma metastásico (figuras 5, 6, 7 y 8).

Con el diagnóstico de neoplasia trofoblástica gestacional tipo coriocarcinoma (BHCG > $100.000 \mathrm{UI} / \mathrm{ml}$ ), metastásico a pulmón, grupo tratante indica manejo sistémico con quimioterapia con cisplatino, etopósido y bleomicina según protocolo.

Respecto al hipertiroidismo, el manejo indicado fue metimazol $15 \mathrm{mg} /$ día y propanolol $40 \mathrm{mg}$ cada 8 horas, además se indica hacer medición cada 15 días de BHCG y T4L para titulación de metimazol. Un mes después del egreso médico, trae reporte de T4L por debajo del rango de referencia $0,68 \mathrm{ng} / \mathrm{dl}$ (VR: 0,8-2 ng/dl) y BHCG en descenso (1123 mUI/ml) por lo que se suspende metimazol.

\section{Discusión}

La tirotoxicosis es el aumento de hormonas tiroideas séricas con consecuencias clínicas; puede ser de origen tiroideo, inflamatorio o extratiroideo. El origen tiroideo es por exceso de producción y liberación de T4L y T3 (hipertiroidismo), cuyas causas incluyen bocio tóxico difuso (enfermedad de Graves), bocio tóxico multinodular (enfermedad de Plummer), adenoma tóxico, y etiologías más raras como hipertiroidismo por estímulo de la HCG, en la enfermedad trofoblástica gestacional (ETG). En cuanto a causas inflamatorias encontramos la tiroiditis, con liberación excesiva de hormona preformada por la glándula; las extratiroideas por ingesta suprafisiológica de tiroxina, entre otras ${ }^{(6)}$.

La subunidad alfa de la HCG (glucoproteína secretada por las células trofoblásticas de la placenta) guarda una homología en su dominio transmembrana ${ }^{(7,8,9,12)}$ con la TSH, la hormona luteinizante y la folículo estimulante ${ }^{(12)}$; por lo que existe una reactividad cruzada entre estas hormonas ${ }^{(8)}$. Sin embargo, la actividad tirotrópica de la HCG es tan solo 1/1.000 en comparación a 1/10.000 la de TSH, por lo que se requieren altos niveles de la misma para generar un cuadro de tirotoxicosis ${ }^{(7)}$.

En cuanto al hipertiroidismo manifiesto (definido como TSH suprimida con elevación de T3 y/o T4L) se requieren niveles de BHCG $>100.000$ para producirlo ${ }^{(8,7,2,10)}$, por tanto, la neoplasia trofoblástica gestacional (mola invasora, coriocarcinoma, tumor trofoblástico del sitio placentario y tumor trofoblástico epitelioide) es una de las causas, y con menor probabilidad, los tumores de células germinales, (ej. tumores
Figura 1. Rx de tórax Opacidades algodonosas (flechas)

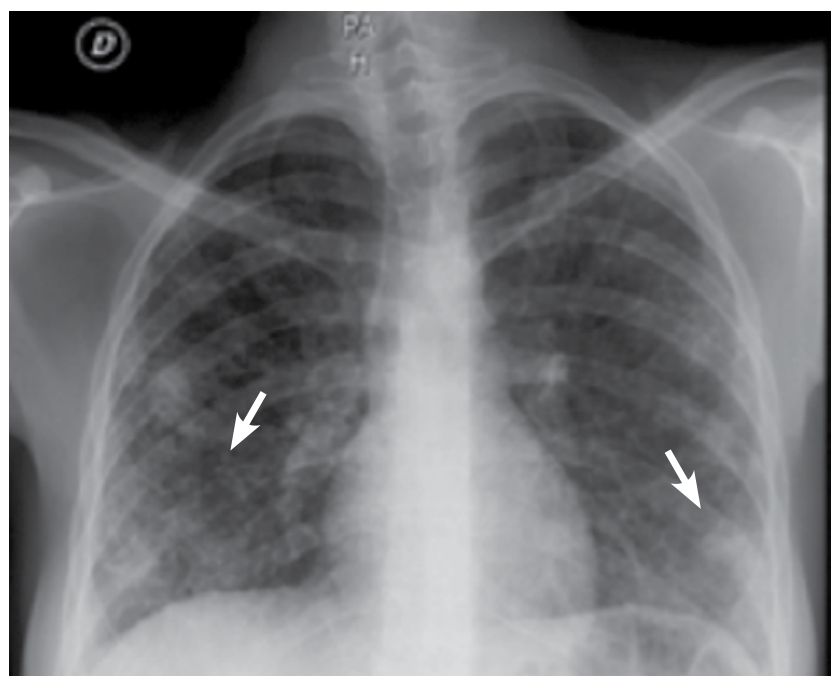

Figuras 2 y 3 . TAC de tórax

2. Corte coronal 3. Corte sagital.

Micronódulos pulmonares en aspecto de árbol de gemación (flechas)
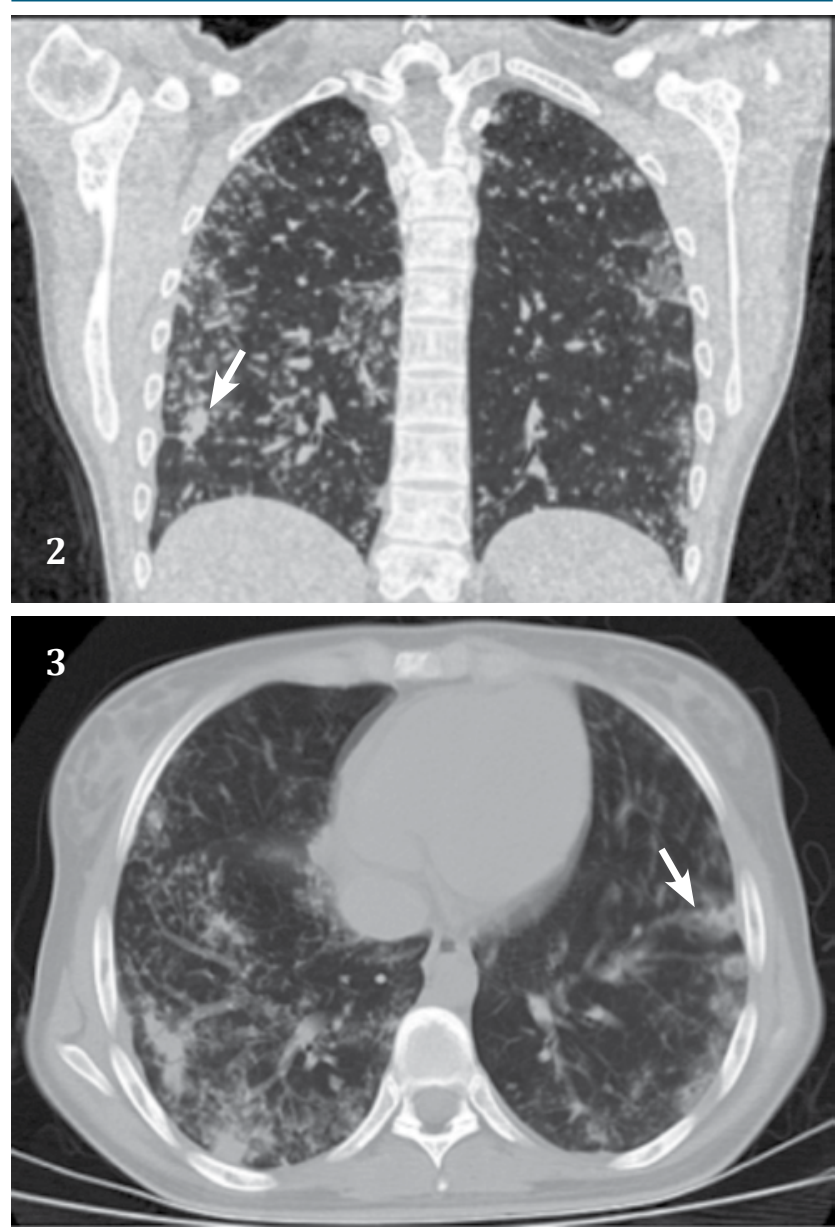
Figura 4. TAC de abdomen

Imágenes quísticas multilobuladas con escasa captación sin componente sólido (flechas)

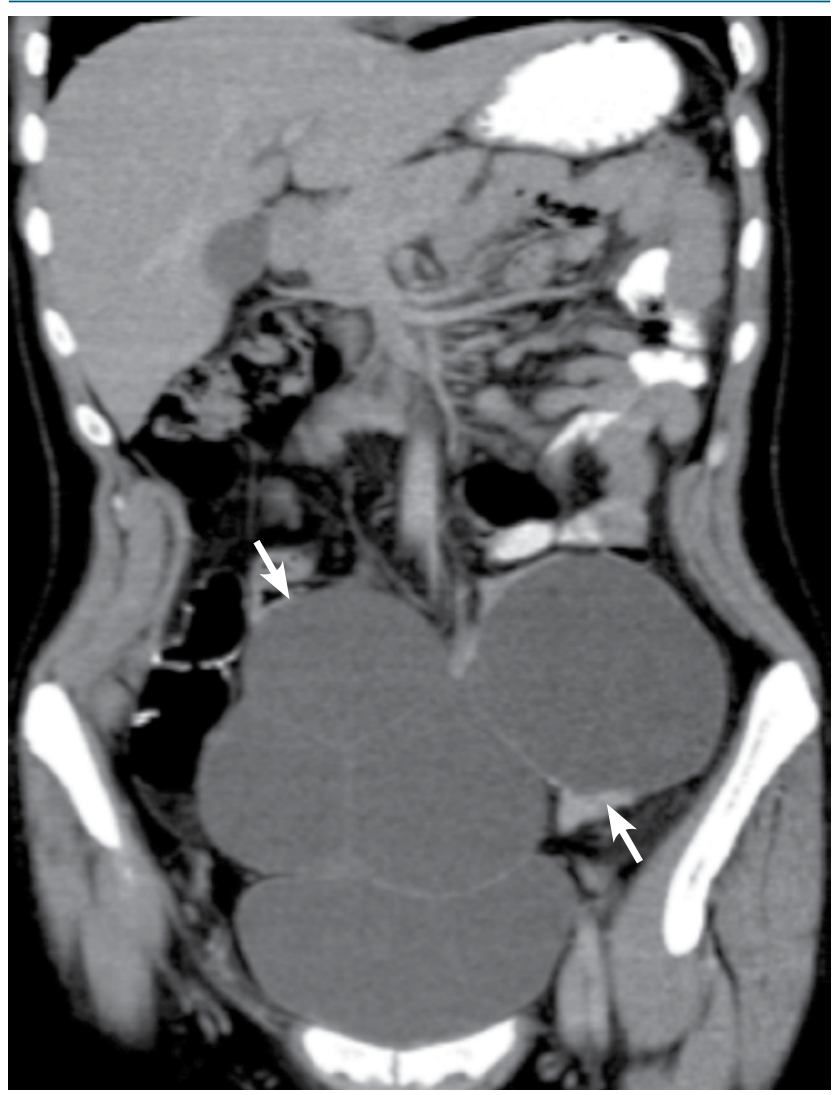

Figura 5. Hematoxilina eosina 4x. Se observa biopsia bronquial con representación de lesión tumor con necrosis

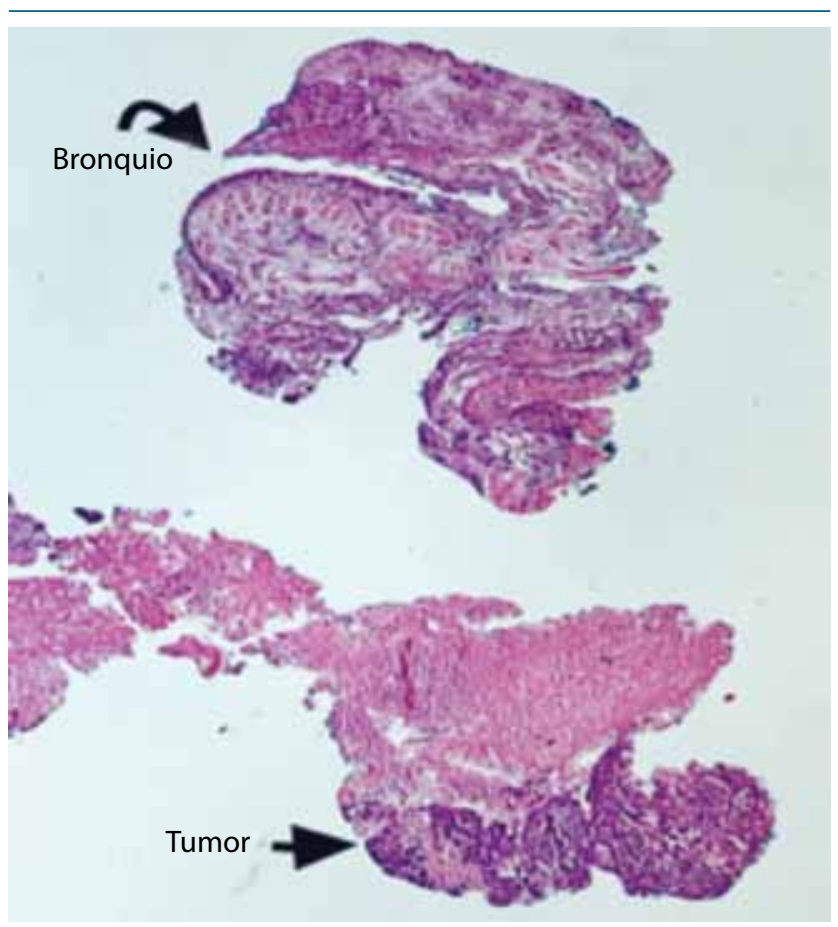

Figura 6. Hematoxilina eosina 40x

Se observa lesión tumoral con células grandes de núcleos pleomórficos hipercromáticos

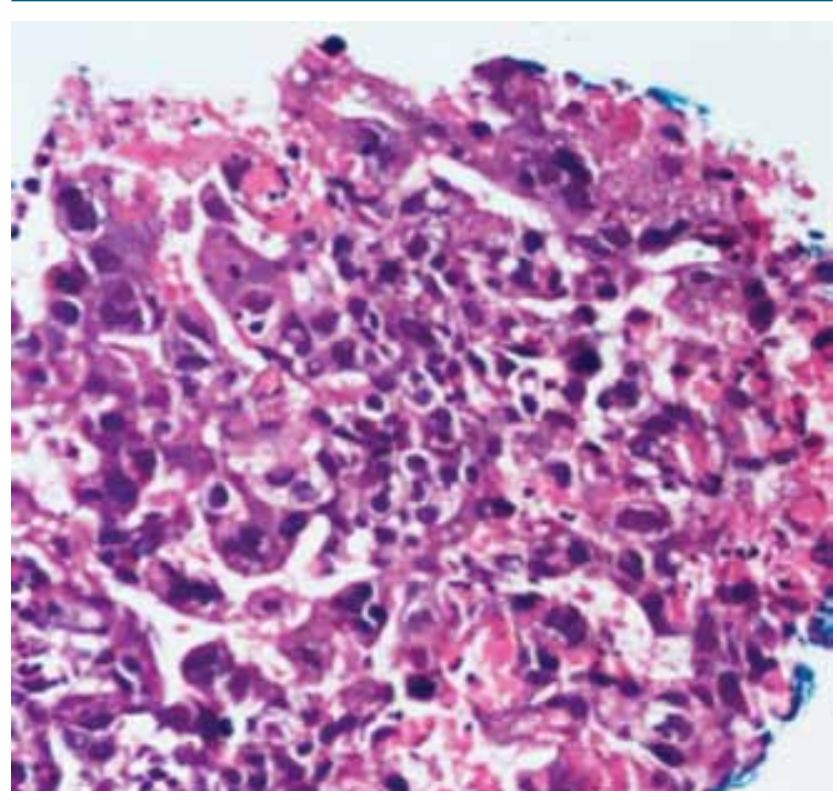

Figura 7.

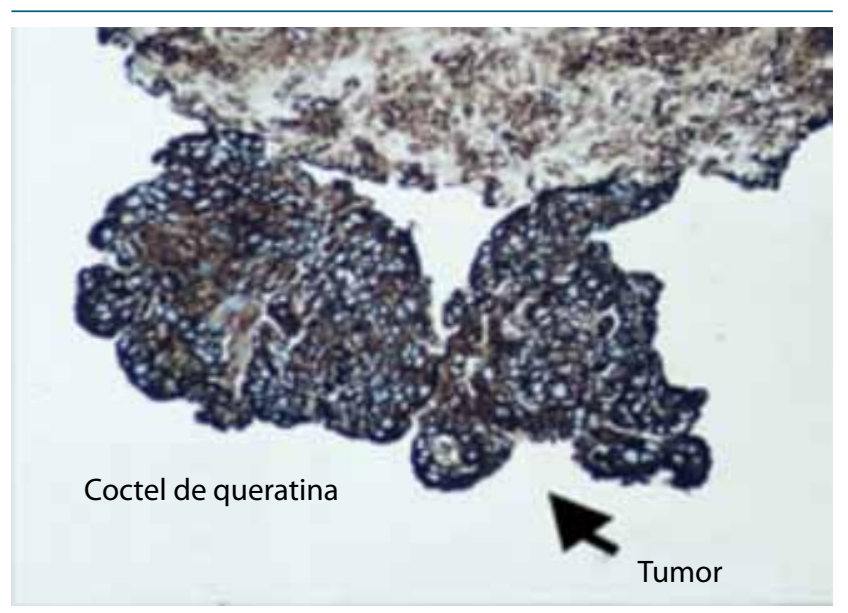

Figura 8.

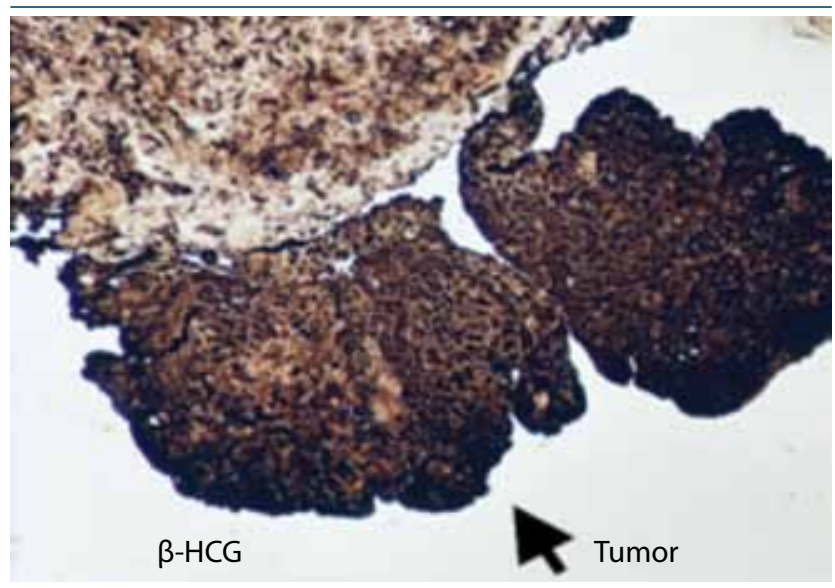

http://revistaendocrino.org/ 
testiculares) como tumores productores de grandes cantidades de BHCG $^{(1,4,8)}$.

La prevalencia de tirotoxicosis en Estados Unidos se estima en $1,2 \%$ ( $0,5 \%$ sintomáticos y $0,7 \%$ subclínicos), de mayor predominio entre los 20 y 50 años de edad, secundarios a la enfermedad de Graves (60\% a 80\% de los casos), mientras que la tiroiditis subaguda corresponde a 15\%-20\%, el bocio multinodular a $10-15 \%$, y el adenoma tóxico a 3\%-5\% ${ }^{(6)}$.

La neoplasia trofoblástica gestacional (NTG) se presenta con mayor frecuencia en Asia que en Norteamérica o Europa, ${ }^{(2)}$. En estas dos últimas áreas, el coriocarcinoma (CCA) afecta aproximadamente a 1/40.000 embarazos, mientras que en el sudeste de Asia y Japón hay una tasa mayor de 9,2 y 3,3 por 40.000 embarazos, respectivamente ${ }^{(5,8,13)}$. El 50\% de los casos de NTG ocurren en gestaciones posmolares (CCA es 1.000 veces más probable después de una mola hidatiforme completa que después de otro tipo de embarazo) $25 \%$ después de abortos o embarazos ectópicos y $25 \%$ en posparto a término o pretérmino ${ }^{(1,4,5)}$. Es más común en mujeres en edad reproductiva ${ }^{(5)}$, se caracterizan por su potencial invasivo y metastásico (pulmón $80 \%$, vagina $30 \%$, cerebro $10 \%$, hígado $10 \%$ ), requiriendo cirugía y/o quimioterapia, a la cual son altamente sensibles con unas tasas de curación superiores al 95\%, con un pronóstico favorable ${ }^{(1,4,11)}$.

Las características clínicas observadas en el hipertiroidismo trofoblástico incluyen fatiga, pérdida de peso, debilidad, diaforesis, nerviosismo, intolerancia al calor, taquicardia, aumento mínimo de la glándula tiroides, sin presencia de oftalmopatía y en casos graves como la tormenta tiroidea - náuseas, vómitos y dolor abdominal. Es frecuente atribuirle estos síntomas a la NTG, pudiéndose pasar por alto el estado hipermetabólico del hipertiroidismo, que puede ocasionar complicaciones clínicas en la paciente, de ahí la importancia de sospecharlo siempre ${ }^{(7,8)}$.

El manejo del hipertiroidismo por NTG generalmente va dirigido a la carga tumoral(8), este se resuelve rápidamente después de la evacuación molar, mientras que la secundaria al CCA tarda más en resolverse ${ }^{(8)}$, por lo cual en estos casos puede ser preferible, además de la quimioterapia, el manejo concomitante para hipertiroidismo, como ocurrió en el caso clínico presentado, donde la paciente estaba francamente sintomática, tenía niveles altos de BHCG, con TSH suprimida y T4L elevada, presentando tirotoxicosis en contexto de un coriocar- cinoma, optándose por la quimioterapia junto con metimazol y propanolol, para desmonte gradual, según respuesta clínica y bioquímica. Dicha conducta se ha realizado previamente en otros centros médicos, según reporte de casos descritos como el Centro de Enfermedades Trofoblásticas de Nueva Inglaterra, donde de 196 casos de NTG tratados con quimioterapia entre 2005 y 2010, 14 (7\%) tenían hipertiroidismo bioquímico y 4 tenían hipertiroidismo clínico (tirotoxicosis) los cuales tuvieron una excelente respuesta al manejo dual, volviendo a la estabilidad metabólico-clínica y al estado eutiroideo, luego de la quimioterapia y el desmonte gradual de antitiroideos y betabloqueadores $^{(8)}$.

\section{Conclusión}

La enfermedad tiroidea bioquímica en pacientes con NTG es relativamente común en Asia, pero en nuestro país no tenemos datos de su incidencia, por tanto, la medición de la función tiroidea es importante en pacientes con este diagnóstico. El hipertiroidismo clínico necesita de una gran carga tumoral y BHCG (>100.000), para manifestarse, siendo menos común y en ocasiones requiriendo tratamiento específico. En aquellos pacientes que requieren tratamiento, el metimazol y los betabloqueadores para el control de la tirotoxicosis y alivio de síntomas parecen ser efectivos, y se podrían suspender una vez que la función tiroidea se normalice luego del tratamiento de la NTG y consiguiente caída de la BHCG. Aun así, no queda claro cuánto tiempo de tratamiento antitiroideo se debe dar con el fin de evitar un hipotiroidismo iatrogénico, tampoco sabemos cómo debería ser la frecuencia de seguimiento clínico y bioquímico del paciente, para lo cual, con base en la literatura y la experiencia de nuestro caso, se requiere vigilancia estrecha, previendo también el riesgo de toxicidad hepática o hematológica del metimazol; desconocemos además el riesgo de que estos pacientes presenten tormenta tiroidea. Se necesitan, por tanto, más estudios y una guía para el manejo de esta condición que, aunque rara, tiene gran impacto en el estado de salud y pronóstico del paciente.

\section{Conflictos de interés Ninguno}

\section{Referencias}

1. Bolze P.-A. et al., Formalised consensus of the European Organisation for Treatment of Trophoblastic Diseases on management of gestational trophoblastic diseases. Eur J Cancer. 2015 Sep;51(13):1725-31.

2. Padmanabhan LD. Et al., Trophoblastic hyperthyroidism. J Assoc Physicians India. 2003 Oct;51:1011-3.

3. N.S. Horowitz, et al., Placental site trophoblastic tumors and epithelioid trophoblastic tumors: Biology, natural history, and treatment modalities, Gynecol Oncol. 2017 Jan;144(1):208-214.

4. Biscaro A. et al., Diagnosis, classification and treatment of gestational trophoblastic neoplasia, Rev Bras Ginecol Obstet. 2015 Jan; 37(1):42-51.

5. Goldstein DP. et al., Current management of gestational trophoblastic neoplasia. Hematol Oncol Clin North Am. 2012 Feb;26(1):111-31.

6. Devereaux D. et al., Hyperthyroidism and thyrotoxicosis. Emerg Med Clin North Am. 2014 May;32(2):277-92.

7. Singh I. et al., Pathogenesis of Hyperthyroidism. Compr Physiol. 2016 Dec 6;7(1):67-79.
8. Walkington L. et al., Hyperthyroidism and human chorionic gonadotropin production in gestational trophoblastic disease. Br J Cancer. 2011 May 24;104(11):1665-9.

9. Lazarus JH. Thyroid function in pregnancy. Br Med Bull. 2011 Dec 23;97:137-48.

10. Fantz CR. Et al., Thyroid function during pregnancy. Clin Chem. 1999 Dec;45(12):2250-8.

11. Monchek R. et al., Gestational trophoblastic disease: an overview. J Midwifery Women's Health. 2012 May-Jun;57(3):255-9.

12. Seckl MJ. Et al., Gestational trophoblastic disease. Lancet. 2010 Aug 28;376(9742):717-29.

13. Yeo CP. Et al., Prevalence of gestational thyrotoxicosis in Asian women evaluated in the 8th to 14th weeks of pregnancy: correlations with total and free beta human chorionic gonadotropin. Clin Endocrinol (Oxf). 2001 Sep;55(3):391-8 